\title{
Experimental Investigations of Cold Flow Mass Distribution and Pressure Drop Characteristics in a Structured Honeycomb Monolith Bed
}

\author{
Mingming Mao, Yongqi Liu*, Ruixiang Liu, Bin Zheng and Jian Meng
}

School of Transportation and Vehicle Engineering, Shandong University of Technology, Zibo 255049, China

\begin{abstract}
The thermal flow reversal reactor (TFRR) and catalytic flow reversal reactor (CFRR) can utilize the ventilation air methane (VAM) from coal mines effectively. The initial cold feed flow mass distribution uniformity in the monolith oxidation bed, the critical part of both reactors, has a great influence on the stability of methane oxidation process and conversation rate. So the emphases are put on the experimental investigations of the cold flow mechanics in the monolith bed based on a simplified reactor test rig. Firstly, the flow resistance coefficients of the monolith bed were calculated, and then the flow resistance mathematical model was built. It was found that the flow mass distribution uniformity was affected by the pressure drop distribution in the monolith bed, and obviously decreased with increasing inlet velocity. The pressure drop distribution was analyzed through a modified momentum equation. The total pressure loss in the lower header was evidently higher than that in the upper header or monolith bed. Additionally, the local losses at the inlet and outlet of the monolith bed were small, so they could be ignored. Finally, the formula of total pressure losses under different flow conditions was established based on the simplified reactor test rig.
\end{abstract}

Keywords: Flow mass distribution uniformity, flow mechanics, flow reversal reactor, monolith oxidation bed, pressure drop, ventilation air methane.

\section{INTRODUCTION}

Considerable amounts of methane are released into the atmosphere with coal-mine ventilation air (VAM) [1, 2]. A single ventilation shaft may discharge several hundred thousand cubic meters of VAM per hour, and although the concentration of methane in these gas streams is rather low (usually below 1 vol. \%), the emissions thus generated pose an environmental threat and lead to the loss of a potential source of energy.

The utilization of VAM containing very low methane is difficult owing to the fact that the air volume is large $\left(600000 \sim 1000000 \mathrm{~m}^{3} / \mathrm{h}\right.$ discharged from a single ventilation shaft) and the methane resource is variable in concentration and flow rate. Majority of the efforts have been focused on the oxidation of methane in ventilation air. Oxidation methods for methane may be classified as thermal and catalytic oxidation based on the kinetic combustion mechanisms. The promising solution seems to be autothermal combustion in reverse-flow reactors [3-7]. As shown in Fig. (1), both the thermal flow reversal reactor (TFRR) and the catalytic flow reversal reactor (CFRR) employ the flow-reversal principle and exchange heat between a solid medium and methane gas to sustain autothermal combustion, then the energy thus produced can be recovered. The main difference between two techniques is whether the catalyst is used to reduce the required operating temperature for methane oxidation.

*Address correspondence to this author at the School of Transportation and Vehicle Engineering, Shandong University of Technology, Zibo 255049, China; Tel. Fax: +86 533 2782616;

E-mails: liuyongqi65@163.com, shandongmao@163.com
The oxidation bed composed of porous medium is the critical part to realize lean methane oxidation and heat recovery, which can be generally classified into three types: honeycomb monolith, packed bed, and fluidized bed $[8,9]$. The honeycomb monolithic-type bed, with many separated tiny channels parallel to flow direction inside, has best characteristics due to very low pressure drop, high geometrical area, and high mechanical strength. The material of the oxidation bed normally uses ceramic for its high specific heat and thermal inertia. To improve the treatability of VAM, the flow cross section and whole volume of the oxidation bed are usually huge, needing large quantities of monolith units arrayed and stacked. In the reversing flow reactor the feed is periodically switched between the two reactor ends using control valves. During the exothermic reaction, the oxidation bed exhibits a heat trap effect to achieve and maintain an enhanced reactor temperature. After many cycles of flow reversal operations, a quasi-steady state may be achieved in which the reactor temperature profile has a maximum value at the center of the oxidation bed and minimum values at two ends. The asymmetry of temperature profiles is of great importance to the effectiveness and stability of the reactor $[5,10]$.

To achieve good asymmetry of temperature profiles in the oxidation bed, several aspects of work need to be done, which mainly include uniform cold feed distribution, initial even temperature field before reaction, symmetric reverse flow operation, etc. So the initial cold feed distribution plays an important role. The inlet feed flow process into the oxidation bed is a typical variable mass flow process and the large cross-flow section improves the difficulty to achieve uniformity. Thus the heat released during methane oxidation is not uniform. On one hand, the overheated part can be 


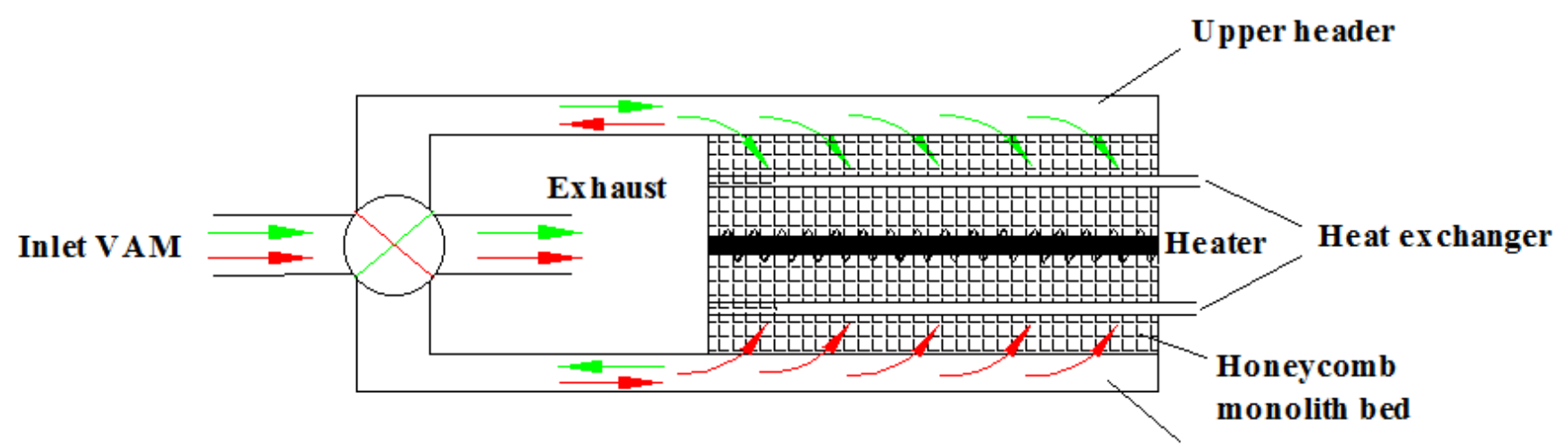

Lower header

Fig. (1). Working principle map of TFRR and CFRR.

affected in lifetime; on the other hand, oxidizing reaction may be terminated in low temperature part. Furthermore, after many cycles of flow reversal operation, the obvious asymmetry of temperature profiles may occur then the stability of methane oxidation reaction process and conversation rate will be seriously affected. So effective measures should be taken to ensure the inlet feed distribution uniformity, such as the use of deflectors. Prior to that, the flow mass distribution characteristics and flow loss mechanism of the monolith bed at different inlet flow conditions ought to be investigated to provide a theoretical guidance for the next step optimization design of intake deflectors.

Currently most of TFRR or CFRR researches are based on numerical simulation and many experimental researches are limited to the investigations of one-dimensional combustion characteristics due to the small-scale reactor test, so there is a real lack of investigations of two-dimensional flow mass distribution and flow pressure loss characteristics of the large cross-sectional structured monolith bed. Therefore, the purpose of this paper is to investigate the flow mass distribution characteristics of a honeycomb monolith bed and pressure loss mechanism on a scaled-down test rig. Because the initial cold feed distribution characteristics have important influences on exothermic oxidation reaction, the involved researches in this paper are totally carried out under cold condition and provide the investigation basis for further study of thermal flow mass distribution characteristics in the thermal reacting system.

\section{EXPERIMENTAL RIG INTRODUCTION}

The experimental setup is shown in Fig. (2a). The external air is blown inside of the test rig through a blower, with the mass flow measured by a rotameter and controlled by a downstream throttling valve. Before entering the honeycomb ceramic monolith bed, the inlet air passes through a separate monolith to even inlet flow. The detailed structure parameters of the monolith bed are illustrated in Fig. (2b). The flow field parameters inside the monolith bed are measured in details. Because of reverse flow symmetry under cold conditions, the flow direction in the ceramic bed remains unchanged. The test rig is designed to have similar flow and same Re range with the practical reverse flow reactor processing VAM volume of $40000 \mathrm{~m}^{3} / \mathrm{h}$.

Fig. (2c) shows the arrangement of measuring points in the test section. Measuring points $1-6,10 \mathrm{~cm}$ apart, are to measure the velocity and pressure distribution along the centerline of monolith bed. From measuring points 7 and 8, $10 \mathrm{~cm}$ apart too, the velocity and pressure drop in the flow direction can be got to calculate the resistance loss coefficients of the monolith bed. Measuring points 9 and 10 , both $10 \mathrm{~cm}$ away from inlet and outlet, are to measure the total pressure drop of the monolith bed and the velocity distribution in vertical direction near inlet and outlet. The throttling valve regulates flow mass entering the reactor test part, ranging $110-270 \mathrm{~m}^{3} / \mathrm{h}$, to obtain corresponding horizontal velocity distribution along the centerline of the monolith bed. As shown in Fig. (2c), the main geometric parameters of the test part are shown in Fig. (2c), with the length $L$ of $0.6 \mathrm{~m}$, width $W$ of $0.2 \mathrm{~m}$ and height $H$ of $0.28 \mathrm{~m}$. The height $h$ of the ceramic monolith bed is $0.2 \mathrm{~m}$, with the porosity $\varepsilon$ of $59 \%$. The upper header and lower header are both $0.04 \mathrm{~m}$ in height $h_{h}$. The measurement errors of various parameters are given as follows: pressure error with a difference-pressure meter is $2 \%$; velocity error with an electronic wind instrument is $2 \%$; flow mass error with a rotameter is $2.5 \%$.

\section{ANALYSIS OF FLOW MASS DISTRIBUTION UNIFORMITY}

\subsection{Flow Mass Distribution in the Monolith Bed}

Fig. (3a) shows the velocity distributions of monolith bed cross section obtained from measuring points 1 to 6 in the different inlet flow conditions. Measuring points 1 to 6 of increasing abscissas get farther from entrance. It is obvious that the velocity throughout the monolith bed becomes higher with larger inlet flow. The velocity gets lower with increasing abscissas of measuring points under various conditions of inlet flow. The trapezoidal velocity distributions are quite uneven.

In order to investigate the effect of each factor on flow mass distribution uniformity, specific evaluation criteria must be given. In this paper, the velocity uniformity index $\gamma$ 
(a) The overall structure



(b) Illustration of the monolith structure

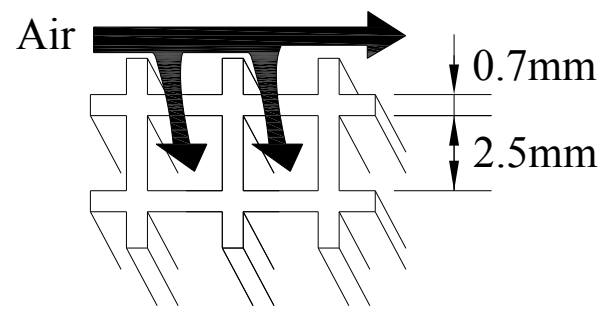

(c) Measuring points in the middle section of the test part

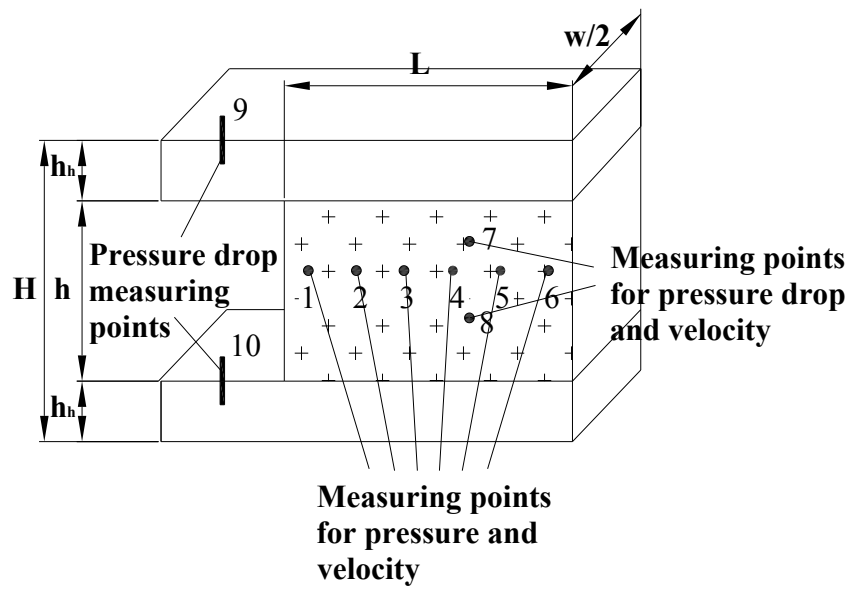

Fig. (2). The experimental setup.

[11] is adopted to value the flow mass distribution uniformity inside the monolith bed. The definition of $\gamma$ is given as follows:

$\gamma=1-\frac{1}{2 n} \sum_{j=1}^{n} \frac{\sqrt{\left(v_{j}-v_{a v}\right)^{2}}}{v_{a v}}$

where $\gamma$ changes between 0 and 1,1 representing ideal uniform flow, 0 indicating extreme uneven flow passing through only one single channel, $v_{j}$ and $v_{a v}$ are respectively the velocity in channel $i$ and average velocity. It's obvious that $\gamma$ can reflex the flow mass distribution situation reasonably. Fig. (4) shows the change curve of velocity uniformity index $\gamma$ with flow mass. It can be seen that the flow mass distribution uniformity decreases with increasing (a) The velocity distributions

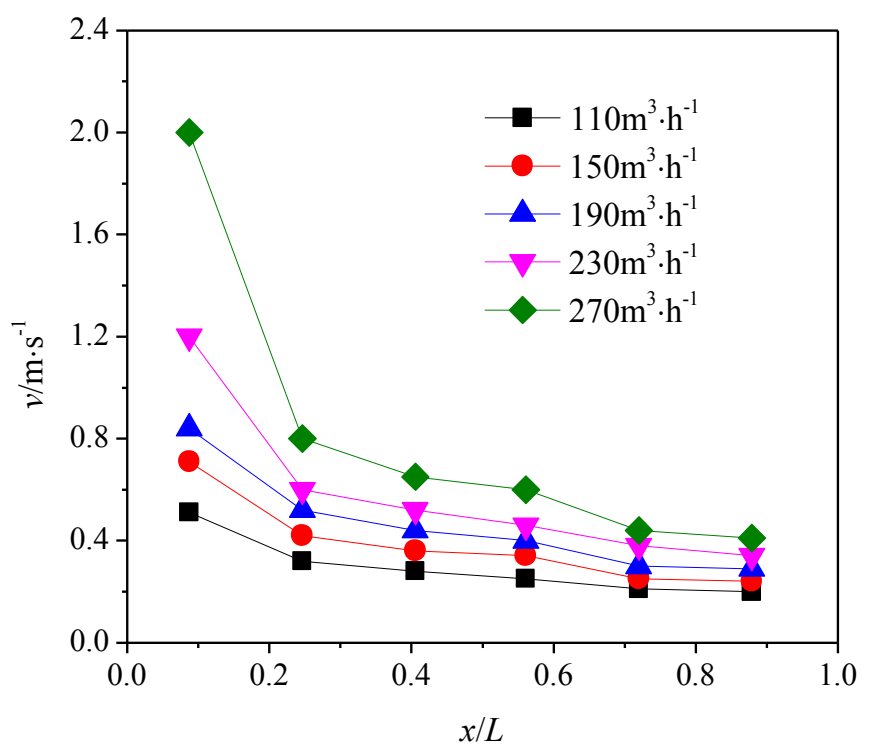

(b) The pressure drop distributions

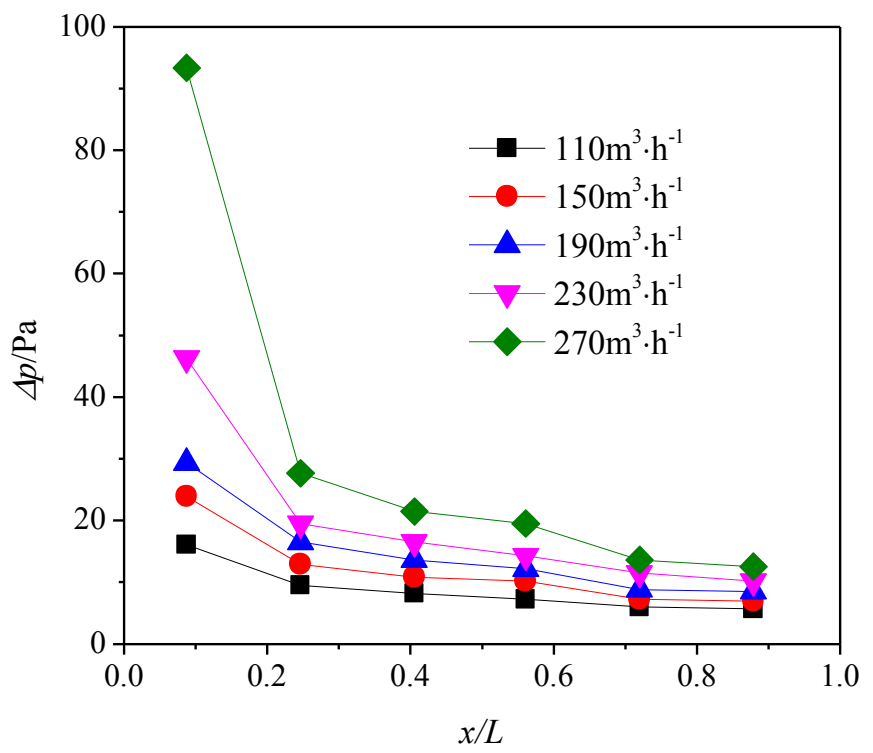

Fig. (3). The velocity and pressure drop distributions of monolith bed bed cross-section at five inlet flow conditions.

flow mass, especially when flow mass exceeds $210 \mathrm{~m}^{3} / \mathrm{h}$. The flow mass velocity uniformity index $\gamma$ tends to slow down when flow mass is lower than $210 \mathrm{~m}^{3} / \mathrm{h}$. In practical application, the flow mass as large as possible should be selected to ensure the handling capacity of VAM on the premise of enough flow mass distribution uniformity.

\subsection{Flow Resistance Mathematical Model of the Monolith Bed}

The air velocity is about 0.2 to $2 \mathrm{~m} / \mathrm{s}$ in the porous monolith bed channels from Fig. (3a), so the corresponding Reynolds number is 34 to 337 . So the internal flow pressure drop is caused by both the viscous resistance and inertial resistance. The relationship of pressure drop and velocity can be described with Darcy-Forchheimer law [12]. That is: 
$\Delta p=\frac{\mu}{k} v \Delta x+\frac{1}{2} c_{F} \rho v^{2} \Delta x$

where $1 / k$ is the viscous resistance coefficient, $k$ is the permeability, $\mu$ is the dynamic viscosity, $v$ is the velocity, $\Delta p$ is the pressure drop, $c_{F}$ is the inertial resistance coefficient, $\rho$ is the density and $\Delta x$ is the flowing distance in a channel of the monolith. According to equation (2), the pressure drop and flow rate can be fit to a quadratic curve. Measured pressure drops from measuring points 7 and 8 at different velocity conditions are shown in Fig. (4) and it's available to get the following fitting equation:

$\Delta p=5.16 v^{2}+13.15 v$



Fig. (4). The change curve of uniformity index with flow mass.

It can be seen in Fig. (5) that the experimental data are very close to the fitting curve. From equation (2) and (3), the viscous resistance coefficient $1 / k$ of $7.6 \times 10^{6}$ and inertial resistance coefficient of 86.65 can be obtained within the monolith bed.

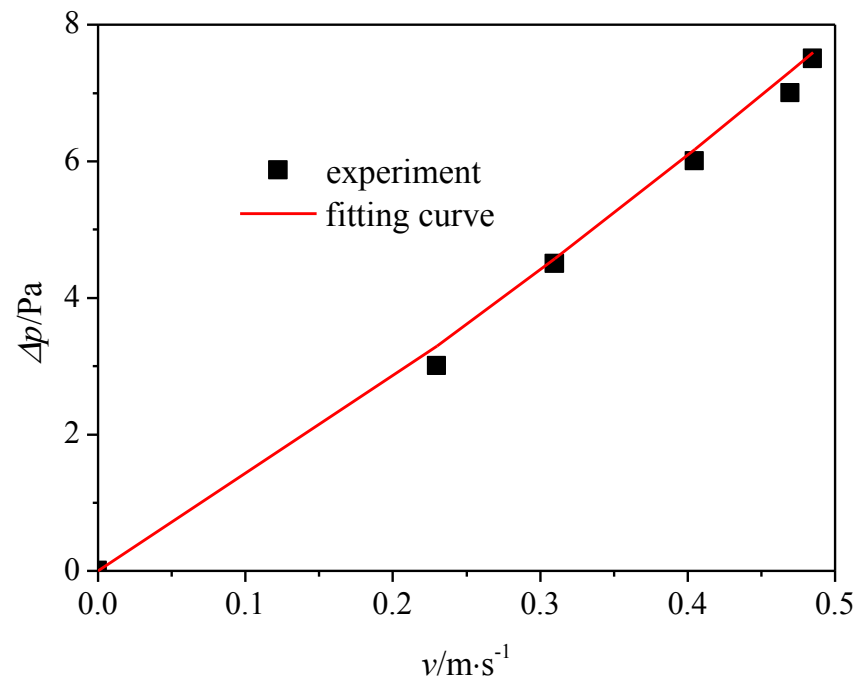

Fig. (5). The change of pressure drop with velocity in the monolith bed.

\subsection{Influencing Mechanism on Flow Mass Distribution Uniformity}

The flow in the channels of the monolith bed is driven by pressure drop between upper header and lower header, so the flow mass distribution uniformity of the monolith bed cross section is closely connected with the distribution situation of pressure drops. According to the velocity distributions in the monolith bed at five flow conditions and equation (2), the responding pressure drops at measuring points 1-6 can be calculated in Fig. (3b). Comparing Fig. (3a, b), the distribution laws of pressure drop and velocity are very similar. Larger pressure drop in the channel near the entrance provides greater driving force leading to higher flow rate.

To clarify the causes of distribution laws of velocity and pressure drop in the monolith bed, fundamental flow mechanism must be analyzed. In the upper header and lower header, continuous importing or separating branch flows along the flow result in variable mass flows. Such flows are generally described with a modified momentum equation [13] as follows:

$\frac{d p}{d z}+2 K \rho u \frac{d u_{m}}{d z} \pm \frac{\lambda \rho u_{m}^{2}}{2 D_{e}}=0$

where "+" before the double symbol term indicates a splitting flow, "-" indicates a gathering flow, $K$ is the momentum exchange coefficient, $\lambda$ is the friction resistance coefficient, $p$ is pressure, $\rho$ is density, $u$ is the mainstream velocity, $D_{e}$ is the equivalent diameter, and $z$ is streamwise coordinate. Equation (4) shows that one part of the static pressure variation along the flow comes from momentum conversion, and another is used to overcome the friction resistance.

As shown in Fig. (6), assume that the inlet static pressure is $p_{u}{ }^{i n}$ and end static pressure is $p_{u}{ }^{e}$ in the upper header, in addition the outlet static pressure is $p_{l}^{o}$ and inner end pressure is $p_{l}^{e}$ in the lower header. According to equation (4), in the upper header the flow mass decreases along the way due to separating branch flows, so the static pressure increases by $\Delta p_{u}{ }^{i}$ for momentum conversion and decreases by $\Delta p_{u}{ }^{d}$ for friction resistance. The total variation of static pressure from import to endpoint is $\Delta p_{u}{ }^{i}-\Delta p_{u}{ }^{d}$ which is relatively small. While in the lower header the flow mass increases along the way due to importing branch flows, so the static pressure decreases for both momentum conversion and friction resistance. The static pressure variation in the lower header is a larger value $\Delta p_{l}^{f}+\Delta p_{l}^{d}$, so the static pressure decreases rapidly. It can be obtained as follows:

$$
\begin{aligned}
& p_{u}^{e}-p_{u}^{i n}=\Delta p_{u}^{i}-\Delta p_{u}^{f} \\
& p_{l}^{e}-p_{l}^{o}=\Delta p_{l}^{f}+\Delta p_{l}^{d} \\
& p_{u}^{e}-p_{l}^{e}-\left(p_{u}^{i n}-p_{l}^{o}\right)=\Delta p_{u}^{i}-\Delta p_{u}^{f}-\Delta p_{l}^{f}-p_{l}^{d}<0
\end{aligned}
$$

It can be seen from (5) that the different variation speeds of static pressure in the upper header and lower header result in greater pressure drop of $p_{u}{ }^{i n}-p_{l}{ }^{o}$ in the monolith bed at the 


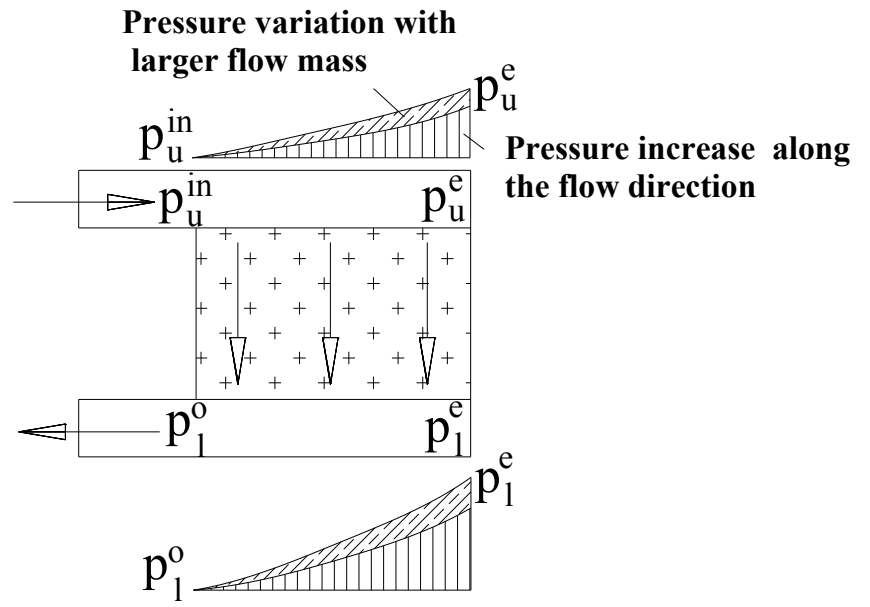

Fig. (6). The static pressure distribution in upper and lower heads.

outer side close to import and export than the pressure drop of $p_{u}{ }^{e}-p_{l}{ }^{e}$ at the inner side. Therefore, the flow mass distribution law of more flow mass at outer side and less at inner side is created.

Song et al. (1992) [14] discovered that the momentum exchange coefficient $K$ is connected with the relative variation of kinetic energy per unit length of mainstream. When the inlet flow mass increases, the kinetic energy per unit length of mainstream and $K$ both increase subsequently, enhancing the effect of momentum exchange on static pressure and causing higher $\Delta p_{u}{ }^{i}$ and $\Delta p_{l}{ }^{d}$. On the other hand, the friction resistance increases in both upper header and lower header with the increase of inlet flow mass and lead to higher $\Delta p_{u}{ }^{d}$ and $\Delta p_{l}^{f}$. As a final result, the gap between the pressure drops at outer side and inner side of monolith bed increases with larger inlet flow mass according to equation (5), so the flow mass distribution becomes more uneven.

\section{ANALYSIS OF TOTAL PRESSURE LOSSES}

\subsection{Compositions of Total Pressure Losses}

The total pressure losses in the test part are mainly composed of five parts, as shown in Fig. (7a). In accordance with the loss mechanism, the total pressure losses can be classified as friction losses and local losses. The friction losses, caused by gas viscosity, exist in the upper header, lower header and the monolith bed. The local losses, present at the inlet and outlet of monolith bed, are generated due to micromass collision and vortex generation because of sudden shrink or expansion of cross-flow area. According to the loss generated location, the overall total pressure loss $\Delta p$ consist of the loss in the upper header $\Delta p_{u}$, loss in the lower header $\Delta p_{l}$ and loss in the monolith bed $\Delta p_{m n}$. Understanding of the calculation method and proportion of each part of total pressure losses has great significance to loss reduction.

The friction losses in the upper header $\Delta p_{u}^{f}$ and lower header $\Delta p_{l}^{f}$ are directly proportional to the fluid dynamic viscosity $\mu$, flow path length $L_{f l}$, flow density $\rho$ and inlet flow velocity $U$ of $\mathrm{mth}$ power, and inversely proportional to the square of flow cross section equivalent diameter, which can be expressed as the following formula:

$$
\begin{aligned}
& \Delta p^{f}=\Delta p_{u}^{f}+\Delta p_{l}^{f} \\
& =C_{u} \mu L_{f l} \rho U^{m_{u}} D_{e}^{-2}+C_{l} \mu L_{f l} \rho U^{m_{l}} D_{e}^{-2}
\end{aligned}
$$

where $m_{u}$ and $m_{l}$ are friction loss experience indexes in the upper and lower header, $C_{u}$ and $C_{l}$ are friction loss experience coefficients in the upper and lower header, which are connected with flow $R e$ and wall roughness.

(a) The five composing parts of total pressure losses in the test part

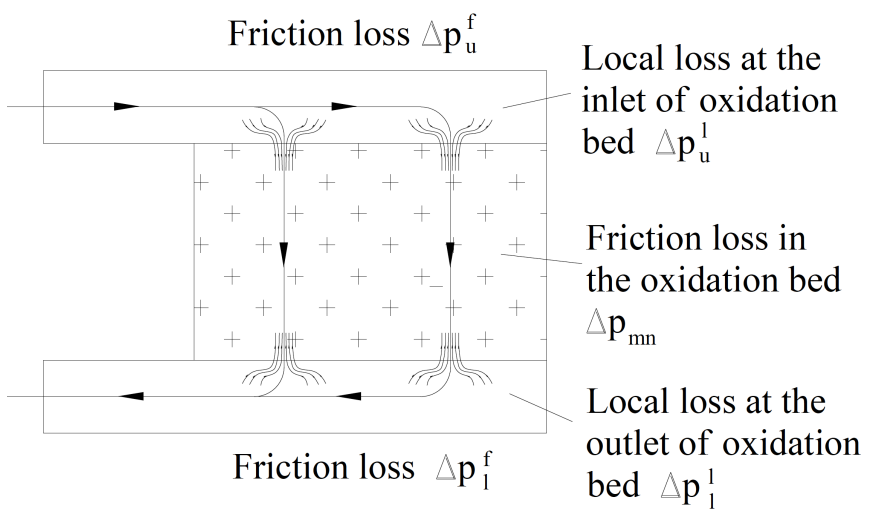

(b) The local flow states at the inlet and outlet of the monolith bed

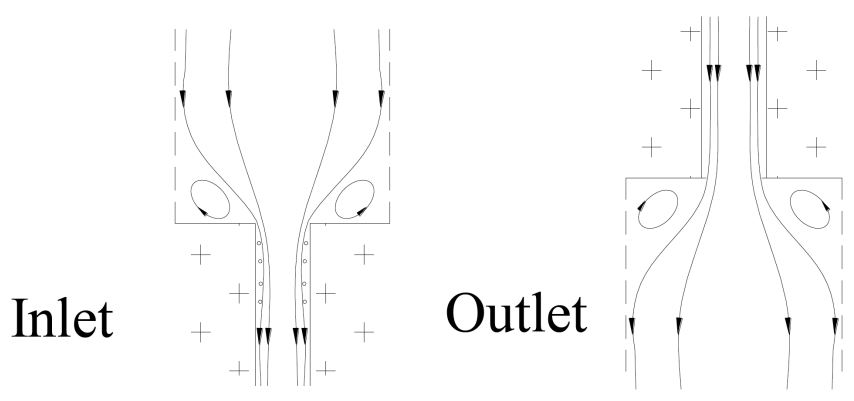

Fig. (7). Sketch map of loss compositions and flow states in the test part.

The local flow states at the inlet and outlet of the monolith bed are shown in Fig. (7b). Many small vortexes are generated in the regions between the mainstream and wall due to sudden variation of cross-flow area and cause flow energy consumption. In order to simplify the problem, the flow through the monolith bed can be decomposed into many single-channel flows with sudden variation of crossflow area at inlet and outlet. The local pressure losses $\Delta p_{j}^{l}$ through a single channel composed of inlet contraction loss $\Delta p_{j}^{i n}$ and outlet diffusion loss $\Delta p_{j}{ }^{\circ}$ can be expressed as follows:

$$
\begin{aligned}
& \Delta p_{j}^{l}=\Delta p_{j}^{i n}+\Delta p_{j}^{o} \\
& =\xi^{i n} \frac{u^{2} \rho}{2}+\xi^{o} \frac{u^{2} \rho}{2}
\end{aligned}
$$

where, $u$ is the flow velocity in the channel, and $\xi^{\text {in }}, \xi^{o}$ are local resistance coefficients at inlet and outlet. The local flow losses at inlet and outlet of the monolith bed can be obtained from the average $\Delta p_{j}^{i n}$ and $\Delta p_{j}{ }^{o}$ values of multiple channels, and then the overall local flow loss $\Delta p^{l}$ can be got. 
Based on experimental studies, the local resistance coefficients $\xi^{i n}$ and $\xi^{o}$ are related to the ratio of branch flow velocity and mainstream velocity. Wang et al. [15-17] obtained the relevant equation of the local resistance coefficient through regression analysis of the experimental data from Song et al. [14], which can be expressed as follows for splitting flow:

$\xi^{\text {in }}=1.446+10^{3} / \exp \left[6.95\left(u / u_{m}\right)^{0.153}\right]$

and for gathering flow:

$\xi^{o}=1.455+4.532 / \exp \left[2.815\left(u / u_{m}\right)^{0.56}\right]$

In the equations (8) and (9), $u_{m}$ is the mainstream velocity.

The pressure drop $\Delta p_{m n}$ in the monolith bed can be calculated according to Darcy-Forchheimer law as follows:

$$
\Delta p_{m n}=h \times\left(\frac{\mu}{k} u+\frac{1}{2} c_{F} \rho u^{2}\right)
$$

In the equation (10), the viscous resistance coefficient $1 / k$ and inertial resistance coefficient $c_{F}$ has been obtained from the pressure drop between measuring point 7 and 8 , so the total pressure loss of the monolith bed can be calculated under given height $h$ and average velocity value $u$.

The overall total pressure loss $\Delta p$ of the test part can be expressed as follows,

$$
\Delta p=\Delta p^{f}+\Delta p^{l}+\Delta p_{m n}
$$

In the equation $\Delta p^{f}$ is difficult to be calculated directly because of the uncertain friction loss experience coefficients $C_{u}$ and $C_{l}$. However, the other kinds of losses including $\Delta p^{l}$, $\Delta p_{m n}$ and $\Delta p$ can be directly calculated easily, so $\Delta p^{f}$ can be obtained in the way subtracting $\Delta p^{l}$ and $\Delta p_{m n}$ from $\Delta p$. Several parts of overall total pressure losses based on two classifications have relationships as follows:

$$
\begin{aligned}
& \Delta p_{u}=\Delta p_{u}^{f}+\Delta p_{u}^{l} \\
& \Delta p_{l}=\Delta p_{l}^{f}+\Delta p_{l}^{l} \\
& \Delta p=\Delta p_{u}+\Delta p_{l}+\Delta p_{m n} \\
& =\Delta p_{u}^{f}+\Delta p_{u}^{l}+\Delta p_{l}^{f}+\Delta p_{l}^{l}+\Delta p_{m n} \\
& =\Delta p^{f}+\Delta p^{l}+\Delta p_{m n}
\end{aligned}
$$

\subsection{Calculation Results of Total Pressure Losses}

The inlet and outlet total pressure distributions of the test part can be obtained from measuring points 9 and 10, then the overall total pressure loss $\Delta p$ can be calculated. The total pressure distribution in the mid of the monolith bed can be measured from points 1-6, so the inlet and outlet total pressure distribution of the monolith bed can be calculated according to equation (2). In various inlet flow conditions, the total pressure losses of three compositions shown in Fig. (8a) are determined using the average total pressure at inlet and outlet of the test part and in the two end sections of the monolith bed. It can be seen from Fig. (8a) that three (a) Three compositions of total pressure losses change with flow mass

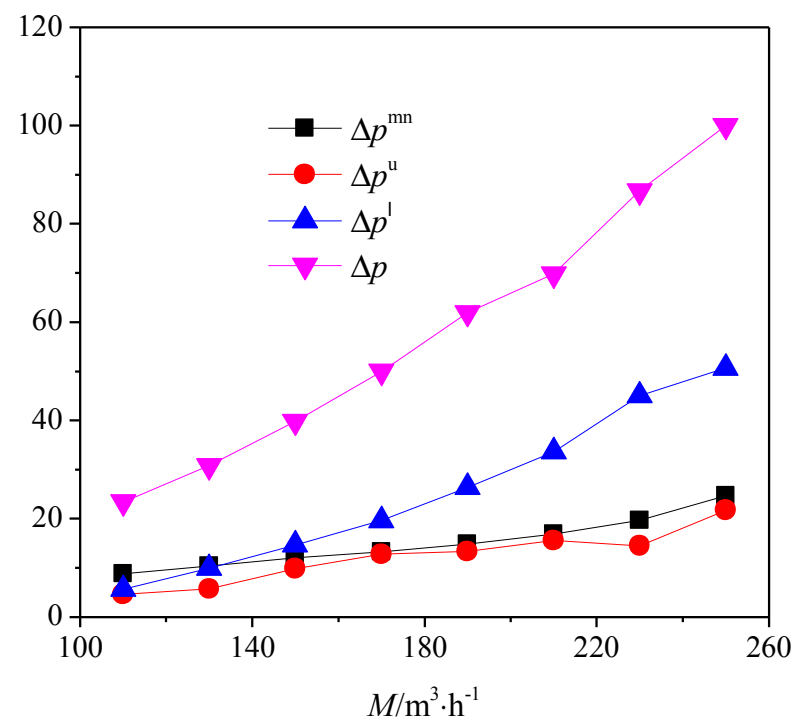

(b) The friction loss in upper and lower headers changes with flow mass

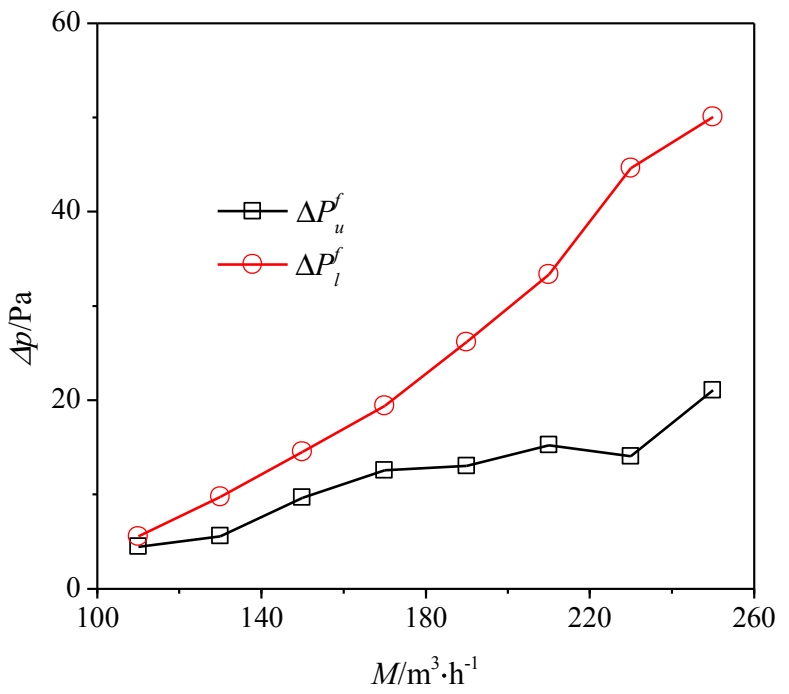

(c) The local loss in upper and lower headers changes with flow mass

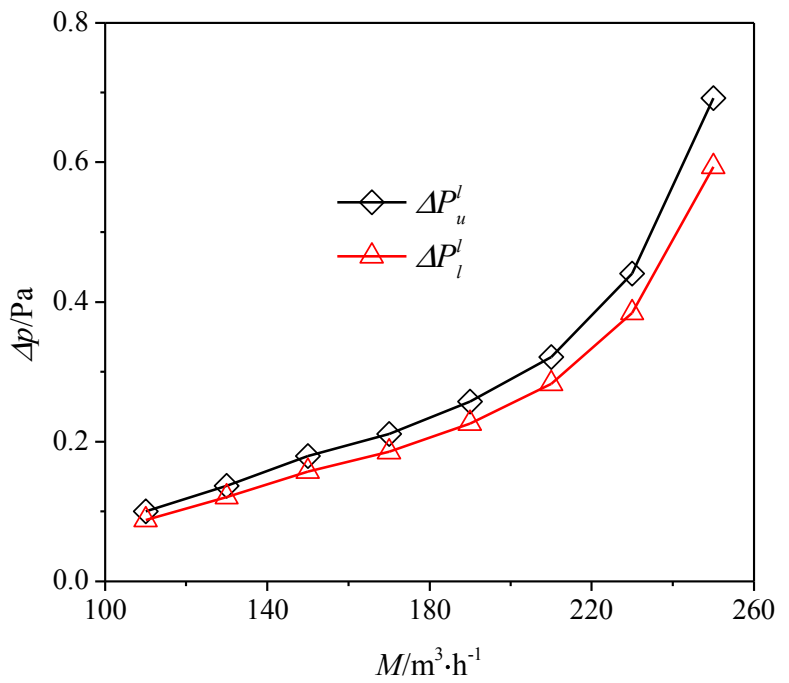

Fig. (8). Change laws of different kinds of total pressure losses with flow mass. 
compositions of total pressure losses all increase obviously with increasing inlet flow mass, because higher velocity leads to more friction losses and local losses. The total pressure loss of the lower header occupies the leading position of three compositions, which is closely related to its flow state.

Fig. (9) shows the vertical distribution at inlet and outlet of the test part in two inlet flow conditions, in which $\mathrm{v}_{1}$ indicates the inlet velocity and $\mathrm{v}_{2}$ indicates the outlet velocity. It can be seen that the distribution of inlet velocity is uniform, however at outlet the velocity at the bottom is significantly higher than on the top. In the lower header, there are continuous branch flows from the channels of the monolith bed converging with the mainstream. Because the direction of branch flows is vertical to that of the mainstream, intense collision and friction occur and consume a part of pressure potential energy to heat. Blocked by the branch flows from the channels, the mainstream flow velocity is low near the outlet section of the monolith bed. So the mainstream flow velocity adjacent to the bottom wall gets higher to maintain the total flow mass, which exacerbates the friction near the bottom wall and causes more friction losses.

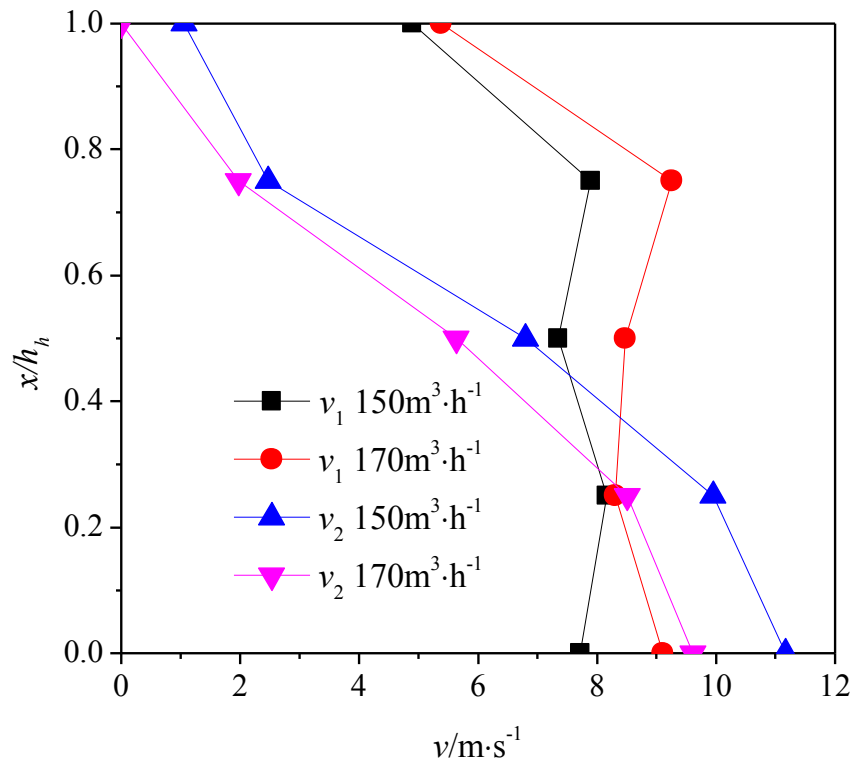

Fig. (9). Vertical distribution of velocity at inlet and outlet of the test part.

In contrast, the branch flows exert much less influences on the mainstream in the upper header and the velocity of mainstream decreases continuously reducing the friction loss near the top wall. In the monolith bed, although the flow velocity is obviously higher than that in the top header and lower header causing more intense friction, the friction loss is the lowest of the three compositions because of the small streamwise length.

Fig. $(\mathbf{8 b}, \mathbf{c})$ shows the friction losses and local losses in the upper and lower header, from which it can be seen that the friction losses obviously dominate total losses. The calculated local losses are no more than $4 \%$ of the friction losses, equivalent to only about $1 \%$ of the total losses of the test part, so the local losses can be ignored to make the losses analysis simplified.

\subsection{Determination of the Pressure Loss Coefficients}

It can be seen from above analysis that the overall total pressure loss of the test part is mainly composed of three parts, namely, the friction losses separately in the upper header $\Delta p_{u}^{f}$ and lower header $\Delta p_{l}^{f}$, and the monolith bed friction loss $\Delta p_{m n}$. The variation laws of the friction losses $\Delta p_{u}^{f}$ and $\Delta p_{l}^{f}$ with inlet flow mass shown in Fig. (8b) can be converted to the rules of $\Delta p_{u}^{f}$ and $\Delta p_{l}^{f}$ changing with inlet velocity, and then transformed into the changes of $\lg \Delta p_{u}^{f}$ and $\lg \Delta p_{l}^{f}$ with $\lg U$ shown in Fig. (10). They can be fitted with linear equations as follows:

$$
\begin{aligned}
& \lg \Delta p_{u}^{f}=1.77 \lg U-0.21 \\
& \lg \Delta p_{l}^{f}=2.67 \lg U-0.57
\end{aligned}
$$

(a) The variation law of $\lg \Delta p_{u}{ }^{f}$ with $\lg U$

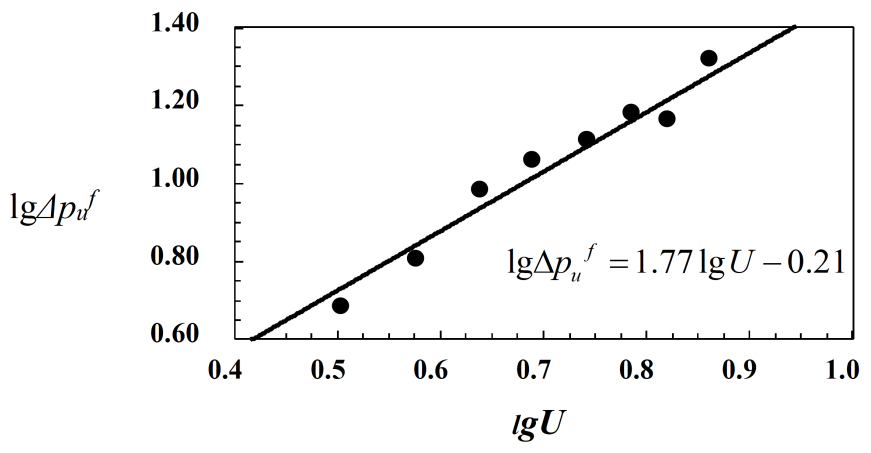

(b) The variation law of $\lg \Delta p_{f}^{f}$ with $\lg U$

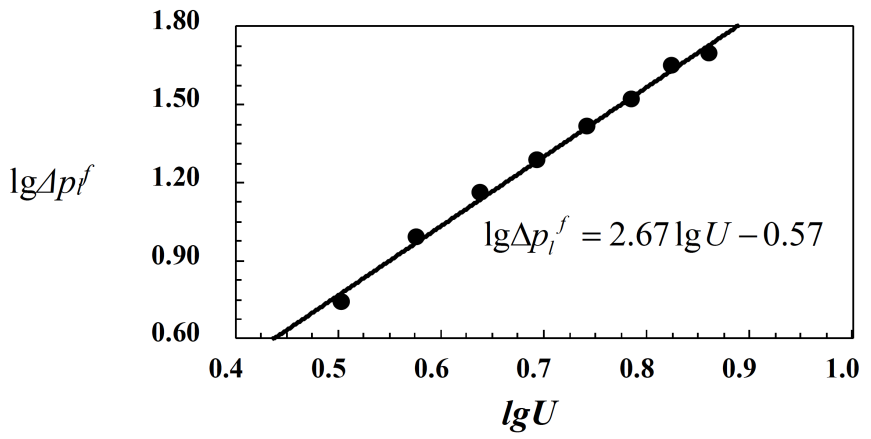

Fig. (10). The variation law of $\lg \Delta p_{u}^{f}$ and $\lg \Delta p_{l}^{f}$ with $\lg U$.

Taking the logarithm on both sides of equation (6), the following formula can be obtained,

$$
\begin{aligned}
& \lg \Delta p_{u}^{f}=m_{u} \lg U+\lg C_{u} \mu L_{f l} \rho D_{e}^{-2} \\
& \lg \Delta p_{l}^{f}=m_{l} \lg U+\lg C_{l} \mu L_{f l} \rho D_{e}^{-2}
\end{aligned}
$$

Comparing equations (13) and (14), the specific values of the frictional experience loss coefficients and frictional experience loss indexes respectively are $C_{u}=213, C_{l}=97$, $m_{u}=1.77, \quad m_{l}=2.67$.

To sum up, the overall total pressure loss of the test part can be calculated as follows: 


$$
\begin{aligned}
& \Delta p=\Delta p_{m n}+\Delta p_{u}^{f}+\Delta p_{l}^{f} \\
& =h \times\left(\frac{\mu}{k} u+\frac{1}{2} c_{F} \rho u^{2}\right)+C_{u} \mu L_{f l} \rho U^{m_{u}} D_{e}^{-2}+C_{l} \mu L_{f l} \rho U^{m_{l}} D_{e}^{-2} \\
& =7.6 \times 10^{6} \times \mu u \times h+86.65 \times \frac{1}{2} \rho u^{2} \times h \\
& +213 \times \mu L_{f l} \rho U^{1.77} D_{e}^{-2}+97 \times \mu L_{f l} \rho U^{2.67} D_{e}^{-2}
\end{aligned}
$$

In the equation (15), the friction loss in the lower header occupying the first place in the total losses is proportional to the 2.67 power of inlet velocity, most sensitive to inlet flow conditions, and therefore increases fastest with increasing inlet flow mass. The equation (15) is obtained under condition that the Reynolds number in the monolith bed ranges between 34 to 337 and the inlet Reynolds number of the test part ranges between 17203 to 42554 . Because The test rig is designed to have similar flow and same Re range with the practical reverse flow reactor processing VAM volume of $40000 \mathrm{~m}^{3} / \mathrm{h}$, equation (15) can be used to calculate the pressure loss of the practical $40000 \mathrm{~m}^{3} / \mathrm{h}$ reverse flow reactor under cold condition. Fig. (11) could validate the accuracy of equation (15) through the comparison the experiment values and calculation values using equation (15) of total pressure losses under different inlet flow mass conditions. It can be seen in Fig. (11) that the calculation values are very close to the experiment values and the maximum difference is no more than $10 \%$ of experiments value.



Fig. (11). The comparison of calculation and experiment values of total pressure loss of the test part.

\section{CONCLUSION}

(1) In the upper header, the static pressure along the flow increases for momentum conversion and decreases for frictional resistance, so the total variation of the static pressure is small. While in the lower header, both momentum conversion and frictional resistance make the static pressure decrease, thus the total variation of the static pressure is comparatively large. The different variation speeds of static pressure in the upper header and lower header result in greater pressure drop in the outer side than the inner side of the monolith bed, which lead to uneven flow mass distribution.

When the inlet flow mass increases, the momentum exchange coefficient and loss coefficient both increase, enhancing the effect of momentum exchange and frictional resistance loss on static pressure. Then the gap between the pressure drops in the outer side and inner side of monolith bed increases, so the flow mass distribution becomes more uneven.

(3) The formula of total pressure losses for the simplified test rig is established and the sensitivity of various losses to the inlet velocity is shown in it. The friction loss in the lower header is most sensitive to inlet flow velocity and therefore increases fastest with increasing inlet flow mass. The overall total pressure loss can be calculated under different flow conditions with this formula.

\section{NOMENCLATURE}

$C_{u} \quad=$ Friction loss experience coefficient in the upper header

$C_{l}=$ Friction loss experience coefficient in the lower header

$D_{e} \quad=$ Equivalent diameter of upper header and lower header (m)

$H \quad=$ Height of the test part $(\mathrm{m})$

$h \quad=$ Height of the monolith bed $(\mathrm{m})$

$h_{h} \quad=$ Height of the upper header and lower header $(\mathrm{m})$

$K=$ Momentum exchange coefficient

$k=$ Permeability $\left(\mathrm{m}^{-2}\right)$

$1 / k=$ Viscous resistance coefficient $\left(\mathrm{m}^{2}\right)$

$L_{f l} \quad=$ Flow path length $(\mathrm{m})$

$L \quad=$ Length of the test part (m)

$m_{u} \quad=$ Friction loss experience index in the upper header

$m_{l} \quad=$ Friction loss experience index in the lower header

$n=$ Total number of channels in the monolith bed

$p \quad=$ Pressure $(\mathrm{Pa})$

$p_{u}{ }^{i n}=$ Inlet static pressure in the upper header $(\mathrm{Pa})$

$p_{u}{ }^{e}=$ End static pressure in the upper header $(\mathrm{Pa})$

$\Delta p_{u}{ }^{d}=$ Static pressure decrease for friction resistance in the upper header $(\mathrm{Pa})$

$\Delta p_{u}{ }^{i}=$ Static pressure increase for momentum conversion in the upper header $(\mathrm{Pa})$

$p_{l}^{o} \quad=$ Outlet static pressure in the lower header $(\mathrm{Pa})$

$p_{l}^{e}=$ End pressure in the lower header $(\mathrm{Pa})$

$\Delta p_{l}^{f}=$ Static pressure decrease for friction resistance in the lower header $(\mathrm{Pa})$ 


$$
\begin{array}{rlrl}
\Delta p_{l}{ }^{d}= & \begin{array}{l}
\text { Static pressure decrease for momentum } \\
\text { conversion in the lower header }(\mathrm{Pa})
\end{array} & \mathrm{o} & =\text { Outlet } \\
\Delta p & =\begin{array}{ll}
\mathrm{f} & =\text { Friction loss }
\end{array} \\
\Delta p_{u} & 1 & =\text { Local pressure loss }
\end{array}
$$$$
\Delta p_{u} \quad=\text { Loss in the upper header }
$$$$
\Delta p_{l} \quad=\text { Loss in the lower header }
$$$$
\Delta p_{m n}=\text { Loss in the monolith bed }
$$$$
\Delta p_{c}=\text { Pressure drop in a channel }(\mathrm{Pa})
$$$$
\Delta p^{f}=\text { The total friction losses in the upper header and }
$$$$
\text { lower header }(\mathrm{Pa})
$$$$
\Delta p_{u}^{f}=\text { Friction losses in the upper header }(\mathrm{Pa})
$$$$
\Delta p_{l}^{f}=\text { Friction losses in the lower header }(\mathrm{Pa})
$$$$
\Delta p^{l}=\text { Local flow losses at inlet and outlet of the }
$$$$
\text { monolith bed }
$$$$
\Delta p_{j}^{l}=\text { Local pressure losses through a single channel } \mathrm{j}
$$$$
\text { (Pa) }
$$$$
\Delta p_{j}^{i n}=\text { Contraction loss at the inlet of a single channel } \mathrm{j}
$$$$
\text { (Pa) }
$$$$
\Delta p_{j}^{o} \quad=\text { Diffusion loss at the outlet of a single channel } \mathrm{j}
$$$$
\text { (Pa) }
$$$$
U=\text { Inlet flow velocity of the test part }\left(\mathrm{ms}^{-1}\right)
$$$$
u=\text { Flow velocity in the channel }\left(\mathrm{ms}^{-1}\right)
$$$$
u_{m}=\text { Mainstream velocity in the upper header or lower }
$$
header $\left(\mathrm{ms}^{-1}\right)$

$v \quad=$ Velocity $\left(\mathrm{ms}^{-1}\right)$

$v_{j} \quad=$ Velocity in channel $\mathrm{j}\left(\mathrm{ms}^{-1}\right)$

$v_{a v}=$ Average velocity $\left(\mathrm{ms}^{-1}\right)$

$W \quad=$ Width of the test part (m)

$\Delta x \quad=$ Flowing distance in a channel of the monolith bed (m)

$z \quad=\quad$ Streamwise coordinates $(\mathrm{m})$

\section{Greeks}

$\gamma \quad=$ Velocity uniformity index

$\varepsilon \quad=$ Porosity of the monolith bed

$\lambda=$ Friction resistance coefficient

$\mu \quad=$ Dynamic viscosity (Pa.s)

$\xi^{i n}=$ Local resistance coefficient at inlet of a single channel

$\xi^{\circ}=$ Local resistance coefficient at outlet of a single channel

$\rho \quad=$ Density $\left(\mathrm{kgm}^{-3}\right)$

\section{Superscripts}

$\mathrm{d}=$ Decrease

e $\quad=$ End

$\mathrm{i}=$ Increase

in $=$ Inlet

\section{Subscripts}

$$
\begin{array}{ll}
\mathrm{av} & =\text { Average } \\
\mathrm{c} & =\text { Channel } \\
\mathrm{e} & =\text { Equivalent } \\
\mathrm{fl} & =\text { Flow } \\
\mathrm{h} & =\text { Header } \\
\mathrm{j} & =\text { Label of a channel } \\
\mathrm{l} & =\text { In the lower header } \\
\mathrm{m} & =\text { Mainstream } \\
\mathrm{mn} & =\text { Monolith bed } \\
\mathrm{u} & =\text { In the upper header }
\end{array}
$$

\section{CONFLICT OF INTEREST}

The authors confirm that this article content has no conflict of interest.

\section{ACKNOWLEDGEMENTS}

The authors gratefully acknowledge the financial supports for this research from the National High Technology Research and Development Program ("863" Program) of China (Grant No. 2009AA063202), Shandong Province Natural Science Foundation Programs of China (Grant No. ZR2011EL017, No. ZR2013EEQ005 and No. ZR2013EEQ008), Shandong Province Science and Technology Development Plan Project of China (Grant No. 2012GGX10417) and 2012 International Cooperation Training Program of Shandong Education Department.

\section{REFERENCES}

[1] O. Y. Rafiu, Z. N. Zainura, H. A. Ahmad, A. A. H. Mohd, and F. M. D. Mohd, "Methane emission by sectors: A comprehensive review of emission sources and mitigation methods", Renewable Sustain Energy Rev., vol. 16, pp. 5059-5070, 2012.

[2] K. Gosiewski, and A. Pawlaczyk, "Catalytic or thermal reversed flow combustion of coal mine ventilation air methane: What is better choice and when?", Chem. Eng. J., vol.238, pp.78-85, 2014.

[3] Y. Liu, J. Han, Y. You, and Z. Gao, "Numerical study on the transient preheating process of a regenerative oxidation bed", Open Mech. Eng. J., vol.5, pp.62-67, 2011.

[4] K. Gosiewski, "Efficiency of heat recovery versus maximum catalyst temperature in a reverse-flow combustion of methane", Chem. Eng. J., vol.107, pp.19-25, 2005.

[5] K. Gosiewski, and K. Warmuzinski, "Effect of the mode of heat withdrawal on the asymmetry of temperature profiles in reverseflow reactors. Catalytic combustion of methane as a test case", Chem. Eng. Sci., vol.62, pp.2679-2689, 2007.

[6] K. Gosiewski, Y. S. Matros, K. Warmuzinski, M. Jaschik, and M. Tanczyk, "Homogeneous vs catalytic combustion of lean methaneair mixtures in reverse-flow reactors", Chem. Eng. Sci., vol.63, pp.5010 -5019, 2008.

[7] K. Gosiewski, A. Pawlaczyk, K. Warmuzinski, and M. Jaschik, "A study on thermal combustion of lean methane-air mixtures: Simplified reaction mechanism and kinetic equations", Chem. Eng. $J .$, vol.154, pp. 9-16, 2009 
[8] M. A. Mujeebu, M. Z. Abdullah, M. Z. Abu Bakar, A. A. Mohamad, R. M. N. Muhad, and M. K. Abdullah, "Combustion in porous media and its applications- A comprehensive survey", $J$. Environ. Manage., vol.90, pp.2287-2312, 2009.

[9] S. Su, and J. Agnew, "Catalytic combustion of coal mine ventilation air methane", Fuel, vol.85, pp.1201-1210, 2006.

[10] S. Salomons, R. E. Hayes, M. Poirier, and H. Sapoundjiev, "Modeling a reverse flow reactor for the catalytic combustion of fugitive methane emissions", Comput. Chem. Eng., vol.28, pp.1599-1610, 2004.

[11] H. G. Tao, H. X. Chen, J. L. Xie, and Y. P. Hu, "An alternative approach to quantifying fluid flow mass distribution uniformity based on area-weighted average velocity and mass-weighted average velocity", Energy Build., vol.45, pp.116-123, 2012.

[12] S. Srinivasan, and K. R. Rajagopal, "A thermodynamic basis for the derivation of the Darcy, Forchheimer and Brinkman models for flows through porous media and their generalizations", Int. $J$. Nonlinear Mech., vol.58, pp.162-166, 2014.
[13] Z. Z. Mu, J. F. Wang, and T. F. Wang, "Optimum design of radial flow moving-bed reactors based on a mathematical hydrodynamic model", Chem. Eng. Processing, vol.42, pp.409-417, 2003.

[14] X. Q. Song, Z. W. Wang, and Y. Jin, "Hydrodynamics of radial flow moving-bed reactor", China Chem. Ind. Eng., vol.43, pp.268274, 1992.

[15] J. F. Wang, X. H. Du, Y. Jin, and Z. Q. Yu, "Hydrodynamic behavior of the gas distribution system in radial flow moving-bed reactor", China Pet. Process Pe., vol.28, pp.47-52,1997.

[16] J. F. Wang, Z. W. Wang, Y. Jin, and Z. Q. Yu, "Simulation of the gas distribution system for a radial flow moving bed catalytic reforming reactor in change state flow", China Pet. Tech., vol.26, pp.598-605,1997.

[17] J. F. Wang, S. Jing, and T. F. Wang, "Mathematical modeling and flow field characteristics of radial flow moving-bed reactors", $J$. Chem. Eng., vol.13, pp.435-440, 1999.

(C) Mao et al.; Licensee Bentham Open.

This is an open access article licensed under the terms of the Creative Commons Attribution Non-Commercial License (http://creativecommons.org/licenses/by-nc/3.0/) which permits unrestricted, non-commercial use, distribution and reproduction in any medium, provided the work is properly cited. 